\section{The effects of intertrial interval on discrimination reversal learning in the pigeon*}

\author{
BEN A. WILLIAMS \\ The Colorado College, Colorado Springs, Colo. 80903
}

Pigeons were trained on an extensive series of color-discrimination reversal problems with an intertrial interval (ITI) of $4 \mathrm{sec}$. They were then shifted to ITI values of 15,30 , and $60 \mathrm{sec}$, with each ITI being used for either 10 or 20 successive reversal problems. Reversal learning proficiency was found to be an inverse function of ITI. Also, learning proficiency within a given ITI increased during training on the 15-sec ITI but decreased during training on the 30 - and 60-sec ITIs.

Various recent experiments have focused upon the possible mechanisms of improvement in successive discrimination reversal learning (SDR). One approach to this question is to determine which variables affect the rate of improvement across successive reversals. A variable of some theoretical significance is the intertrial interval (ITI). If substantial improvement in SDR learning occurs only with short ITIs, for example, the range of possible mechanisms to explain SDR improvement would be considerably delimited. To date, however, the effect of ITI on SDR learning remains confused. Three experiments have shown SDR learning to be facilitated by shorter ITIs (North, 1959; Stretch, McGonigle, \& Morton, 1964; Johnson, 1968), whereas three others have shown no effect of ITI (North, 1950a, b Cronholm, Warren, \& Hara, 1960). The purpose of this experiment, therefore, is to extend the investigation of the ITI variable.

Previous experiments have generally presented different ITIs to different groups of Ss, with the separate groups receiving relatively few reversals. Several questions remained unanswered by this procedure, however. For example, if ITI effects do occur, will they be maintained over successive reversals, thus producing different asymptotic levels of SDR improvement, or will the ITI differences gradually disappear? Similarly, will the performance of Ss trained on short ITIs deteriorate immediately when shifted to longer ITIs, and vice versa, or will the Ss continue performing as they did

*This experiment was conducted when the author was a NSF graduate fellow at Harvard University and was supported by Research Grants NIMH 15495 and NIH-GM-15258 to Harvard University. Reprints can be obtained from the author, Department of Psychology, The Colorado College, Colorado Springs, Colo. 80903. before the shift in ITI values? To answer these questions, the present experiment varies the ITI for Ss already extensively trained on a series of discrimination reversal problems in order to determine how ITI affects the asymptotic level of performance. SUBJECTS

The Ss were six White Carneaux pigeons, maintained at $80 \%$ of their free-feeding body weights.

\section{APPARATUS}

The apparatus was an operant conditioning chamber converted from an aluminum picnic cooler. The bird's chamber was a $30.5-\mathrm{cm}$ cube and was equipped with an overhead houselight and a constant white-noise input to mask outside noise. On the front panel, two Gerbrands pigeon keys, $1.9 \mathrm{~cm}$ in diam, were mounted $10 \mathrm{~cm}$ apart, center to center, and $23 \mathrm{~cm}$ off the floor, with each key requiring an application of at least $0.15 \mathrm{~N}$ for operation. Behind each pigeon key were two 7.5-W Christmas tree light bulbs, one of which was illuminated behind each key on each trial. Each peck on either of the keys produced a click resulting from the activation of a relay located just behind the front panel. Directly between and $13 \mathrm{~cm}$ below the keys was a $5.0 \times 4.5 \mathrm{~cm}$ aperture, through which the birds were fed when a food magazine was activated. The electrical programming equipment for the conditioning chamber was located in the next room.

The pigeons were extensively trained on a series of daily discrimination reversals, using a 4 -sec ITI. They received a total of 30 reversals using the stimuli red and yellow and a total of 80 reversals using the stimuli blue and green. This training was presented to establish the asymptotic level of SDR learning proficiency. Within a daily problem, each of two colors was present on each trial. When the $S$ responded to the correct color, the lights were PROCEDURE extinguished and the bird was rewarded with $2.8-\mathrm{sec}$ access to the food magazine; reward was followed by complete darkness for the remainder of the ITI, whereupon the lights came on for the start of the next trial. When the $S$ responded to the incorrect color, the lights went out for the entirety of the ITI. Before a given trial was designated as completed, either 15 pecks to the correct color or 15 pecks to the incorrect color were required. Correct and incorrect pecks were counted separately, so that whichever first reached the FR 15 requirement determined whether the trial was correct or incorrect. The positions of the colors changed randomly after a correct trial but did not change after an incorrect trial. Thus, after an incorrect trial, the bird could use the position last responded to as an additional cue for the next trial.

After the preliminary training, using the 4-sec ITI, the six Ss were divided into two groups of three each to assess the effect of varying the ITI during continued training on the blue-green reversal problem. No changes in procedure were made, except for the ITI. The order of ITI presentation for Group 1 was 10 sessions using a 4 -sec ITI, 20 sessions using a $60-\mathrm{sec}$ ITI, 10 sessions using a 4-sec ITI, 20 sessions using a 15-sec ITI, and 10 sessions using a $30-\mathrm{sec}$ ITI. The order of ITI presentation was the same for Group 2, except that the 15- and 60-sec ITIs were reversed. Throughout this training, the color designated correct for a given session was alternated between sessions, with training in a particular session continuing until the $S$ had reached the criterion of 10 consecutively correct trials.

\section{RESULTS}

Figure 1 shows the mean errors to criterion for reversal learning under each ITI for each S. Because of the correction procedure used, only errors after a preceding correct trial are presented. Two facets of the data are noteworthy: the general level of errors for different ITIs and the way in which reversal learning improved or worsened as a function of training on the same ITI. If the latter point is considered first, it is seen that the changes occurring within an ITI condition vary with the ITI. Ss from both groups decrease their errors to criterion with continued training on the 15-sec ITI, whereas their reversal learning deteriorates with continued training on the 60 -sec ITI (with the exception of $S 1$ in Group 1). For the 30-sec ITI, reversal learning worsens for four of the Ss and stays the same for two others (Ss 1 and 40). 


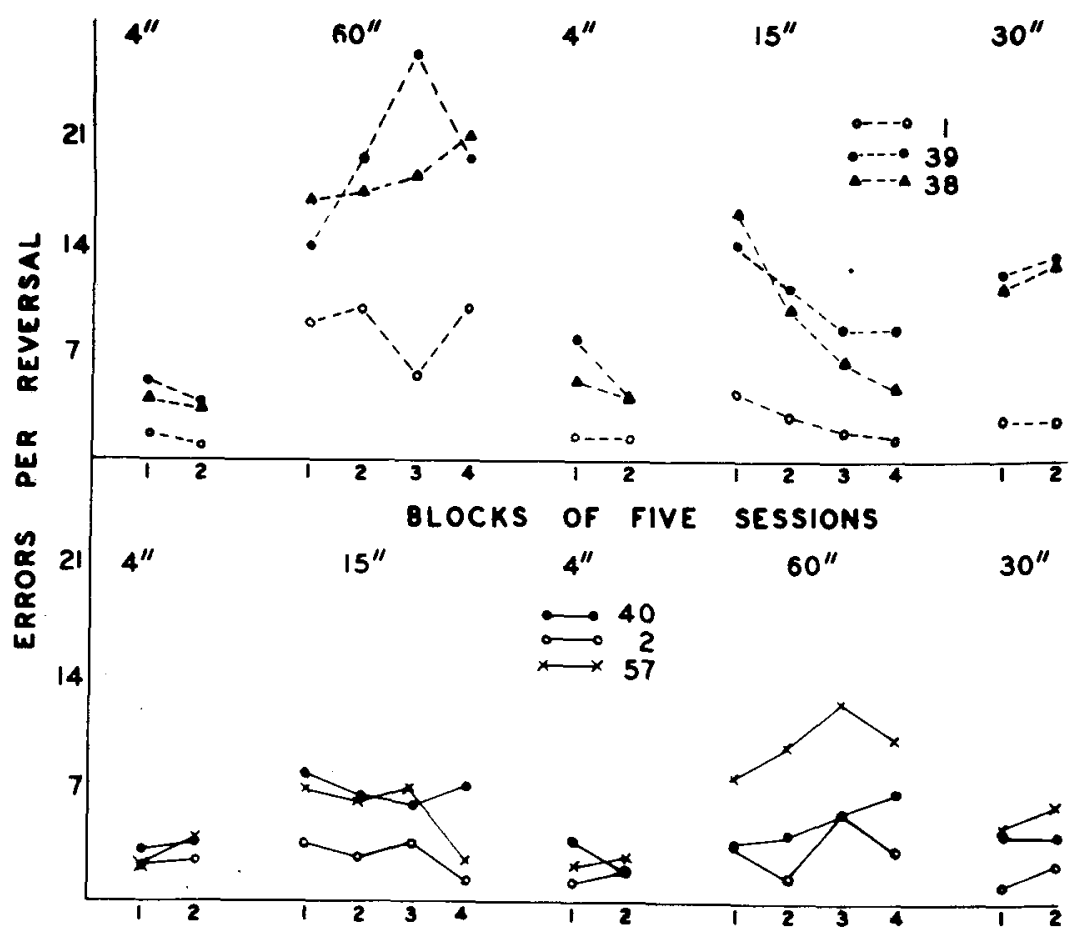

Fig. 1. Mean number of errors to criterion for each $\mathbf{S}$ on each ITI condition. Only errors after a preceding correct trial are presented.

Because the changes occurring within a given ITI condition varied with the ITI used, any question of the overall level of errors as a function of ITI is impossible without considering from which point in training the measure of reversal learning proficiency is taken. Perhaps the best measure possible is to examine the asymptotic performance for each ITI value. Unfortunately, the data shown in Fig. 1 are probably not all at their asymptotic level. Because the changes occurring within ITI conditions are generally monotonic, however, an approximation to this level can be made by taking the performance levels at the end of training on each ITI. If each $S$ is examined individually, it is seen that all Ss in Group 1 show a monotonic relation between ITI and errors to criterion. With the exception of S 40, Group 2 reveals a similar result, although the reversal learning proficiency of these Ss is generally much less sensitive to the ITI variable.

To obtain a more precise quantitative relation between ITI and learning proficiency, it is necessary to equate the level of training on each ITI condition. Since the $30-$ sec ITI was presented for only 10 sessions, such a measure was computed by taking the mean errors to criterion for Sessions 9 and 10 for each ITI condition. These data were averaged across all six Ss and are shown in Fig. 2, with errors after obtained in the present experiment between ITI and rate of SDR learning is in substantial agreement with several previous studies examining the ITI variable (see introduction). In addition, the present experiment shows that the ITI effects persist over continued SDR training, with different ITI values apparently maintaining different asymptotic levels of learning proficiency. Further, the present study shows that ITI effects early in training may have a large transient component, e.g., the 15-sec ITI, which may disappear with further training. This last point is noteworthy in that many experiments examining ITI effects have used only a single reversal problem.

Given the highly regular ITI effects obtained in this experiment, some rationale is needed for the conflicting results of previous SDR experiments varying ITI. If these experiments are considered as a whole, two basic differences among them are apparent. The first is the actual ITI values used. Two of the experiments showing ITI effects used short ITIs (Johnson, 1968: $2.5 \mathrm{sec}$ vs $13 \mathrm{sec}$; Stretch et al, 1964: $0 \mathrm{sec}$ vs $12 \mathrm{~min}$ ), while two of the experiments showing no ITI effect compared relatively long ITIs (North, $1950 \mathrm{a}, \mathrm{b}$ both compared $1 \mathrm{~min}$ vs $12 \mathrm{~min}$ ). The present experiment indicates that much of the ITI effect occurs over the range of $0-60 \mathrm{sec}$. Unless an ITI value below $60 \mathrm{sec}$ was used, therefore, no ITI effects would necessarily appear. There are, however, two exceptions to this generalization. First, North (1959) found that a comparison of a 20 -min ITI with a 24-h ITI yielded significant differences in favor of the shorter value. Secondly, Cronholm et al (1960) compared a

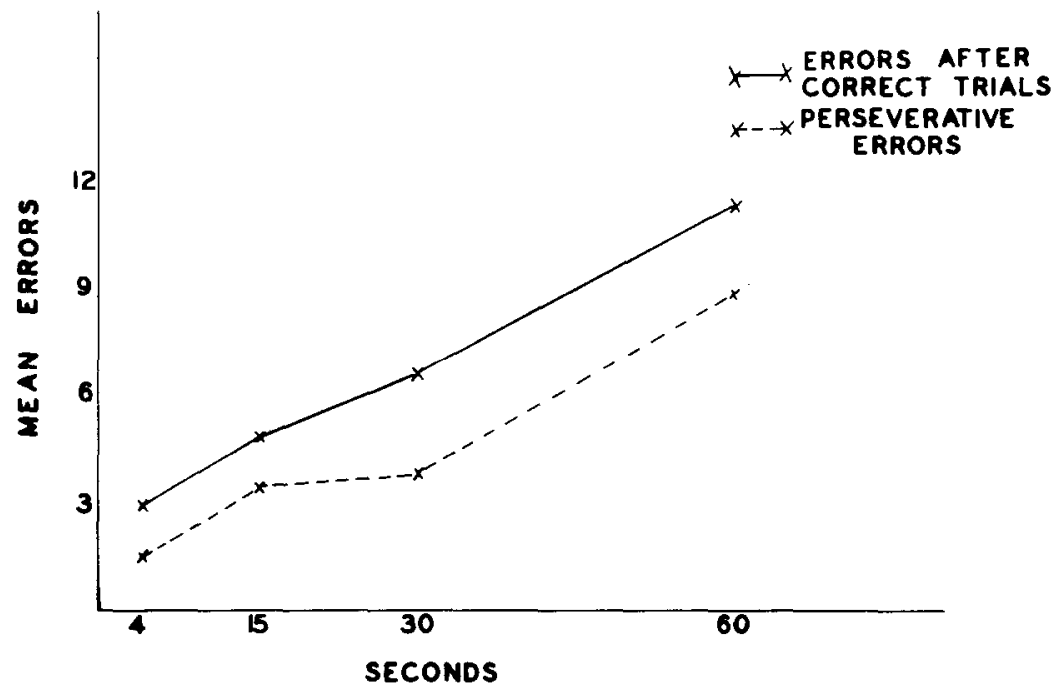

Fig. 2. Mean number of errors averaged over all Ss for sessions 9 and 10 on each ITI condition. Total errors are subdivided into errors after a preceding correct trial and errors after a preceding error (which allowed the use of a position cue). 
10-sec ITI with a 70-sec ITI and found no differences. While the first study may be discounted because intervals as long as $24 \mathrm{~h}$ may have interfering properties, e.g., warm-up effects, quite separate from the usual causes of ITI effects, the second discrepant finding does require some explanation. One possible explanation comes from the type of learning problem involved. Whereas three of the four experiments showing an ITI effect, including the present study, involved visual discriminations, all of the experiments showing no ITI effect involved spatial discriminations. This distinction could possibly explain the Cronholm et al results, which confounded visual and spatial cues, in that their Ss were run in a WGTA and could perhaps have adopted some kind of postural mediation of the delay between trials.

One implication of the present results that must be emphasized is the role of ITI in determining asymptotic performance for SDR learning. As noted above, extended training on the longer ITIs did not yield further improvement in the errors to criterion, in spite of the fact that performance was far from optimal. Since the rate of error reduction in SDR learning is often claimed as an index of comparative intelligence, such comparative assessments must take into account one more procedural variable. Moreover, control for such a variable is greatly complicated by the possibility that the function relating ITI and SDR learning proficiency has different parameters for different species. An adequate control would thus have to determine the ITI function for each type of $\mathrm{S}$. Without such control, the claim that the SDR procedure yields genuine species differences in learning must remain suspect.

\section{REFERENCES}

CRONHOLM, J. N., WARREN, J. M., \& HARA. K. Distribution of training and reversal learning by cats. Journal of Genetic Psychology, 1960, 96, 105-113. JOHNSON, R. N. Effect of intercranial reinforcement intensity and distributional variables on brightness reversal learning in rats. Journal of Comparative \& Physiological Psychology, 1968, 66, 422-426.

NORTH, A. J. Improvement in successive discrimination reversals. Journal of Comparative \& Physiological Psychology, $1950 a, 43,442-460$.

NORTH, A. J. Performance under an extended series of discrimination reversals. Journal of Comparative \& Physiological Psychology, 1950b, 43 , 461-470.

NORTH, A. J. Discrimination reversal with spaced trials and distinctive cues. Journal of Comparative \& Physiological Psychology, 1959, 52, 426-429.

STRETCH, R. G.. MCGONGLE, B., \& MORTON, A. Serial position reversal learning in the rat: Trials/problem and the intertrial interval. Journal of Comparative \& Physiological Psychology, $1964,57,461-463$.
(Bower, 1961). The ceiling effect refers to the possibility that the control group is running so fast to the large reward that the group shifted from small to large reward is incapable of running faster than the control. At least two successful methods for dealing with the ceiling effect have been used. Delay of reward results in running speeds which are slower than nondelay procedures, and positive contrast has been shown to be reliable using the delay procedure (Mellgren, 1970a, b; Shanab, Sanders, \& Premack, 1969). The use of a low drive condition has also been successful in allowing positive contrast to emerge (Marx, 1969; Shanab \& Ferrell, 1970), presumably because performance would be less than maximal due to the low degree of motivation.

Campbell, Crumbaugh, Knouse, \& Snodgrass (1970) attempted to deal with the ceiling effect problem by shifting Ss after relatively few small-reward trials, before the control group reached an asymptote. They were only partially successful in that the control group appeared to reach an asymptote around the 14th trial of the experiment. The experimental groups were shifted after 5,10 , or 15 trials and may have been subject to the ceiling effect. The groups shifted after 5 trials did show a positive contrast effect, but it was not statistically reliable.

The present experiment was designed to explore the possibility that positive contrast may result in the straight runway when very few preshift trials are given. Thus, a total of zero, two, or four trials with small reward preceded the shift to large reward. In addition, a relatively small intertrial interval was used, since this procedure has resulted in very abrupt changes in performance in past studies (Mellgren, 1970b).

\section{METHOD}

A total of 29 male albino rats of the Sprague-Dawley strain were used. The Ss were 80 days old at the start of the experiment.

A 23-h food-deprivation schedule began 8 days before the start of the experiment. During this time all rats were handled for $5 \mathrm{~min}$ each day, and their tails were marked. On the 2 days immediately before the start of the experiment, all Ss were brought to the experimental room and distributed into individual cages but had no experience in the runway.

The runway was constructed of wooden walls painted medium gray, hinged ceilings made of clear Plexiglas, and a grid floor made of transverse stainless steel bars, $10 \mathrm{~mm}$ in diam, spaced $19.05 \mathrm{~mm}$ on center. The alley section of the runway was $1.219 \mathrm{~m}$ 